\title{
Effectiveness of Plasma and Urine Neutrophil Gelatinase-Associated Lipocalin for Predicting Acute Kidney Injury in High-Risk Patients
}

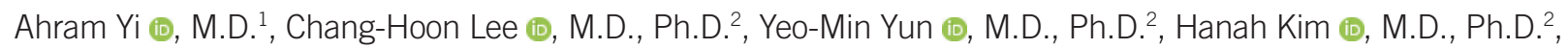 \\ Hee-Won Moon (i), M.D., Ph.D. ${ }^{2}$, and Mina Hur (i), M.D., Ph.D. ${ }^{2}$ \\ ${ }^{1}$ Department of Laboratory Medicine, Green Cross Laboratories, Yongin, Korea; ${ }^{2}$ Department of Laboratory Medicine, Konkuk University School of Medicine, \\ Seoul, Korea
}

Background: Neutrophil gelatinase-associated lipocalin (NGAL) is a useful biomarker for acute kidney injury (AKI) prediction. However, studies on whether using both plasma NGAL (PNGAL) and urine NGAL (UNGAL) can improve AKI prediction are limited. We investigated the best approach to predict AKI in high-risk patients when using PNGAL and UNGAL together.

Methods: We enrolled 151 AKI suspected patients with one or more AKI risk factors. We assessed the diagnostic performance of PNGAL and UNGAL for predicting AKI according to chronic kidney disease (CKD) status by determining the areas under the receiver operating curve (AuROC). Independent predictors of AKI were assessed using univariate and multivariate logistic regression analyses.

Results: In the multivariate logistic regression analysis for all patients ( $N=151$ ), Model 2 and 3, including PNGAL ( $P=0.012)$ with initial serum creatinine $(\mathrm{S}-\mathrm{Cr})$, showed a better AKI prediction power $\left(R^{2}=0.435\right.$, both) than Model 0 , including $S-C r$ only $\left(R^{2}=0.390\right)$. In the non-CKD group ( $N=135)$, the AuROC of PNGAL for $A K I$ prediction was larger than that of UNGAL $(0.79$ vs $0.66, P=0.010)$, whereas in the CKD group $(N=16)$, the opposite was true (0.94 vs $0.76, P=0.049)$.

Conclusions: PNGAL may serve as a useful biomarker for AKI prediction in high-risk patients. However, UNGAL predicted AKI better than PNGAL in CKD patients. Our findings provide guidance for selecting appropriate specimens for NGAL testing according to the presence of CKD in AKI high-risk patients.

Key Words: Plasma, Urine, Neutrophil gelatinase-associated lipocalin, Acute kidney injury, Chronic kidney disease
Received: October 19, 2019

Revision received: February 10, 2020

Accepted: July 29, 2020

\section{Corresponding author:}

Chang-Hoon Lee, M.D., Ph.D.

Department of Laboratory Medicine, Konkuk University School of Medicine, Konkuk University Chungju Hospital, 82 Kukwondae-ro, Chungju 27376, Korea Tel: +82-43-840-8381

Fax: +82-43-840-8967

E-mail: chlee@kku.ac.kr

\section{Co-corresponding author:}

Yeo-Min Yun, M.D., Ph.D.

Department of Laboratory Medicine, Konkuk University School of Medicine, Konkuk University Medical Center,

120-1 Neungdong-ro, Gwangjin-gu,

Seoul 05030, Korea

Tel: +82-2-2030-5582

Fax: +82-2-2030-5587

E-mail: ymyun@kuh.ac.kr

\section{(i) (3)}

(c) Korean Society for Laboratory Medicine This is an Open Access article distributed under the terms of the Creative Commons Attribution Non-Commercial License (https://creativecommons.org/licenses/by-nc/4.0) which permits unrestricted non-commercial use, distribution, and reproduction in any medium, provided the original work is properly cited.

\section{INTRODUCTION}

The Kidney Disease: Improving Global Outcomes (KDIGO) Clinical Practice Guidelines for Acute Kidney Injury (AKI) defines $\mathrm{AKI}$ according to the serum creatinine (S-Cr) concentration [1].
However, the role of S-Cr as a kidney function marker is limited because its concentration is influenced by multiple non-kidney factors, including age, sex, muscle mass and metabolism, dietary habits, medications, and hydration status. Moreover, the half-life of S-Cr increases from four hrs to 24-72 hours, if the 
baseline glomerular filtration rate (GFR) falls [2-5]. Therefore, a true decrement in GFR may not be appropriately reflected by the $\mathrm{S}-\mathrm{Cr}$ concentration in patients with sepsis, liver disease, and/or muscle wasting [2-4]. Baseline S-Cr concentrations are already high in patients with chronic kidney disease (CKD), regardless of an AKI event, leading to difficulty in discriminating AKI. Thus, there is a need for a reliable AKI biomarker that is sensitive, specific, and easily measured to predict early changes following kidney injury without the need for exogenous substance administration [6]. Early AKI prediction is especially crucial to prevent AKI development or progression in high-risk patients who are older, have sepsis, are affected by multiorgan failure, have preexisting chronic comorbidities, and/or are taking nephrotoxic drugs, which can be a cause of AKI [7].

As an alternative to $\mathrm{S}-\mathrm{Cr}$, neutrophil gelatinase-associated lipocalin (NGAL) is a useful biomarker for AKI prediction [8-12]. Plasma NGAL (PNGAL) and urine NGAL (UNGAL) were previously measured using separate conventional immunoassays [13-17]. Recently developed assays enable the measurement of both PNGAL and UNGAL using the same reagent in routine chemistry analyzers. However, there is still no consensus on the type of specimen (blood or urine) that is most appropriate for NGAL assays in the initial work-up for AKI prediction; PNGAL concentrations might be influenced by extra-kidney factors and that elevated UNGAL concentrations provided a more specific estimation of kidney tubular damage [18]. Blood collection is routinely performed to test several laboratory parameters; the same specimens could be used for PNGAL tests [18]. By contrast, urine collection is difficult in incontinent patients, requiring bladder catheterization, which is an invasive procedure. Despite the need for a comparison between PNGAL and UNGAL for the AKI prediction in high-risk patients, few studies have evaluated the diagnostic performances of PNGAL and UNGAL tests simultaneously in patients without CKD, admitted to an intensive care unit (ICU) or a critical care setting and in oliguric, critically ill patients without CKD [19-21].

Therefore, there is a need to explore PNGAL and UNGALbased approaches for AKI prediction and attempt to reach a consensus regarding the most appropriate assay. We evaluated the AKI prediction powers of PNGAL and UNGAL in AKI highrisk patients with and without CKD. We also assessed whether PNGAL and UNGAL measurements add value to S-Cr measurement for predicting AKI in AKI high-risk patients. To our knowledge, this is the first study to investigate PNGAL and UNGAL measurements simultaneously for predicting AKI in AKI highrisk patients mainly based on patients visiting the emergency department, especially with CKD.

\section{MATERIALS AND METHODS}

\section{Subjects and specimens}

We enrolled patients for whom the NGAL assay was recommended for suspected AKI at Konkuk University Medical Center, Seoul, Korea, between October 2016 and April 2017. In total, 200 patients were enrolled in this retrospective study. After excluding 49 patients without S-Cr data to determine AKI and/or CKD status, a total of 151 AKI high-risk patients were included (140 from the emergency department, nine from the obstetrics and gynecology department, two from the internal medicine department, and one from the neurosurgery department). High risk for AKI was determined based on the presence of one or more AKI risk factors, such as old age ( $>65$ years old), sepsis, chronic preexisting chronic comorbidities (e.g., diabetes mellitus, hypertension, chronic heart disease, and CKD), and intake of nephrotoxic drugs (e.g., antibiotics, iodinated contrast agents, nonsteroidal anti-inflammatory drugs, and anticancer agents) [7, 22]. AKI was defined based on the KDIGO clinical practice guidelines [1]; the initial and serial S-Cr and estimated-GFR (eGFR) data of every patient were reviewed and applied. Urine output criteria were excluded. The patients with eGFR below $60 \mathrm{~mL}$ $\mathrm{min} / 1.73 \mathrm{~m}^{2}$ for more than 90 days were considered to have CKD. This study was approved by the Institutional Review Board (KUH1200071) of Konkuk University Medical Center. This registry study required neither study-specific blood or urine collection nor other interventions. Therefore, the requirement of written informed consent from the patients was exempted.

A total of 151 residual specimens (EDTA plasma and urine) were consecutively collected immediately after the onset of suspected clinical manifestations of AKI. The specimens were divided into small aliquots to avoid repeated freezing and thawing and were stored at $-70^{\circ} \mathrm{C}$ until use. Frozen specimens were thawed at room temperature $\left(23^{\circ} \mathrm{C}\right.$ to $\left.24^{\circ} \mathrm{C}\right)$ and gently mixed just before biomarker measurement. The PNGAL and UNGAL concentrations were measured between March and June 2017.

\section{Assays}

PNGAL and UNGAL concentrations were simultaneously measured using a particle-enhanced turbidimetric immunoassay (The NGAL Test, Bioporto Diagnostics A/S, Hellerup, Denmark) with a TBA-C16000 instrument (Toshiba Co., Tokyo, Japan). The analytical measurement range of the NGAL test is $25-3,000 \mathrm{ng} /$ $\mathrm{mL}$ for both plasma and urine. The total coefficient of variation 
Table 1. Distribution of the study population according to AKI risk factors $(\mathrm{N}=151)$

\begin{tabular}{|c|c|}
\hline Susceptibility factor & $N(\%)$ \\
\hline Female & $80(53.0)$ \\
\hline Black & $0(0)$ \\
\hline CKD & $16(10.6)$ \\
\hline \multicolumn{2}{|l|}{ Chronic diseases } \\
\hline Heart & $39(25.8)$ \\
\hline Lung & $66(43.7)$ \\
\hline Liver & $11(7.3)$ \\
\hline Diabetes mellitus & $37(24.5)$ \\
\hline Hypertension & $75(49.7)$ \\
\hline Cancer & $40(26.5)$ \\
\hline Anemia & $90(59.6)$ \\
\hline$>65$ yr old & $103(68.2)$ \\
\hline \multicolumn{2}{|l|}{ Exposure } \\
\hline Sepsis & $36(23.8)$ \\
\hline Critical illness* & $151(100)$ \\
\hline Circulatory shock & $14(9.3)$ \\
\hline Burns & $0(0)$ \\
\hline Trauma & $6(4.0)$ \\
\hline Cardiac surgery (especially with CPB) & $11(7.3) / 3(2.0)$ \\
\hline Major noncardiac surgery & $44(29.1)$ \\
\hline \multicolumn{2}{|l|}{ Nephrotoxic drugs } \\
\hline Antibiotics & $30(20.0)$ \\
\hline Radiocontrast agents & $3(2.0)$ \\
\hline NSAIDs & $7(4.6)$ \\
\hline Anticancer drugs & $25(16.6)$ \\
\hline Pesticides & $1(0.7)$ \\
\hline \multicolumn{2}{|c|}{ Overall number of risk factors per patient } \\
\hline 1 & $1(0.7)$ \\
\hline 2 & $6(4.0)$ \\
\hline 3 & $14(9.3)$ \\
\hline 4 & $20(13.2)$ \\
\hline 5 & $19(12.6)$ \\
\hline 6 & $34(22.5)$ \\
\hline 7 & $27(17.9)$ \\
\hline 8 & $16(10.6)$ \\
\hline 9 & $7(4.6)$ \\
\hline 10 & $6(4.0)$ \\
\hline 11 & $1(0.7)$ \\
\hline
\end{tabular}

${ }^{*}$ Critical illnesses include neurological disorders (e.g., stroke, Alzheimer's disease), cancer, heart diseases, and kidney diseases, among others. Abbreviations: AKI, acute kidney injury; CKD, chronic kidney disease; CPB, cardiopulmonary bypass; NSAIDs, nonsteroidal anti-inflammatory drugs.

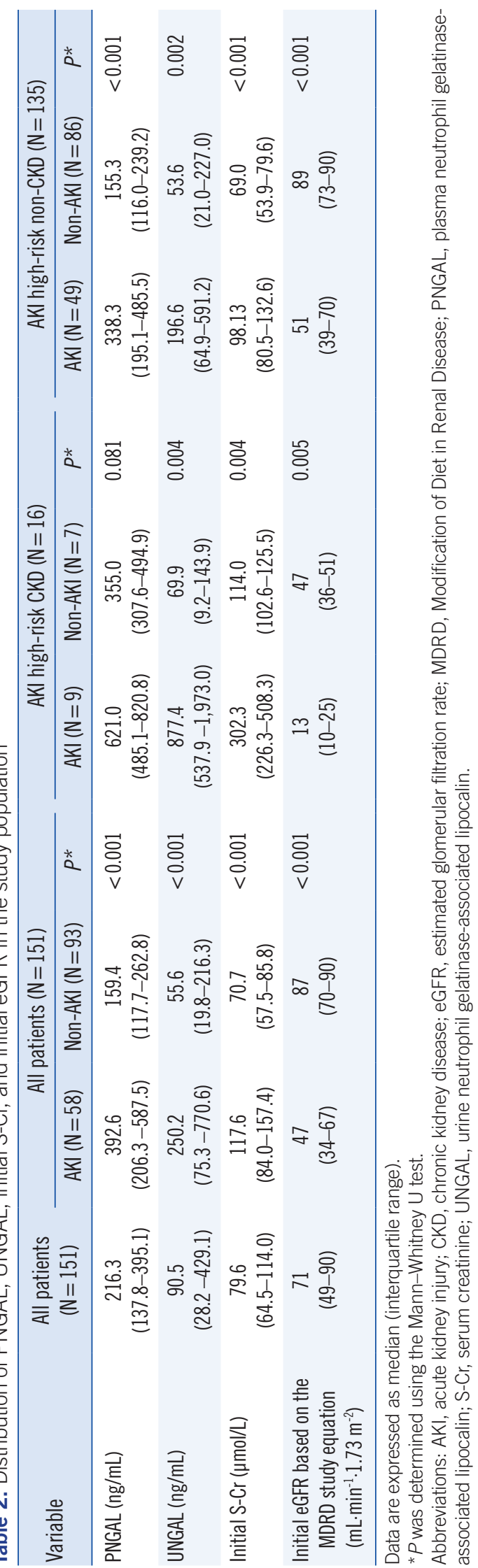


(CV\%) during the study period was $<3.3 \%$ for controls (from The NGAL Test Control Kit ST003CA), urine, and plasma specimens according to the manufacturer.

\section{Statistical analysis}

Data are expressed as median (interquartile range) or number (percentage). Areas under the receiver operating characteristic curves (AuROCs) and their 95\% confidence intervals (Cls) were analyzed to assess the diagnostic performances and optimal cut-off values of PNGAL and UNGAL to predict AKI in all patients and according to the CKD status. PNGAL and UNGAL concentrations were compared between groups created according to clinical events and outcomes, such as AKI, in-hospital mortality, ICU admission, and need for renal replacement therapy (RRT), using the Mann-Whitney $U$ test. Univariate and multivariate logistic regression analyses were performed to assess the predictors of AKI development. Variables with univariate $P<0.05$ (age and kidney biomarkers) were entered into the model for multivariate logistic regression analysis. The MedCalc Statistical Software (version 17.4.4, MedCalc Software bvba, Ostend, Belgium) was used for statistical analyses. $P<0.05$ was considered statistically significant.

\section{RESULTS}

\section{Patient characteristics}

The characteristics of the patients according to AKI risk factors, PNGAL and UNGAL concentrations, initial S-Cr concentration, and initial eGFR are summarized in Tables 1 and 2. All patients had at least one AKI risk factor, demonstrating the diversity of AKI high-risk factors in this population. The AKI and non-AKI groups showed statistically significant differences in the concentrations of PNGAL, UNGAL, and initial S-Cr, and in initial eGFR
$(P<0.05$ for all, Table 2). The same factors differed for patients with and without AKI when stratified by CKD status, except that patients with CKD $(\mathrm{N}=16)$ did not differ in PNGAL concentration from those without CKD (Table 2).

\section{Multivariate logistic regression analysis of risk factors to predict AKI development}

Four models were analyzed by multivariate logistic regression (Table 3). In Model O (age and initial S-Cr concentration) and Model 1 (age, initial S-Cr, and UNGAL), only initial S-Cr concentration was a significant predictor of AKI. In Model 2 (age, initial $\mathrm{S}-\mathrm{Cr}$, and PNGAL) and Model 3 (age, initial S-Cr, PNGAL, and UNGAL), initial S-Cr and PNGAL were significant predictors of $A K I$. The $R^{2}$ value was the highest overall for Model 3. Therefore, initial S-Cr $(P<0.001)$ and PNGAL $(P=0.013)$ concentrations, but not the UNGAL concentration, were considered independent predictors of AKI development in AKI high-risk patients, and the combination of these two factors increased the predictive power of the model.

\section{Performance of PNGAL and UNGAL to predict AKI development and the effect of CKD}

The predictive value of PNGAL and UNGAL concentrations for AKI in AKI high-risk patients is shown in Fig. 1 based on the AuROC values. For all patients, the AuROC for PNGAL was higher than that for UNGAL; there was moderate sensitivity and high specificity for a PNGAL cut-off concentration of $306.1 \mathrm{ng} / \mathrm{mL}$ and moderate sensitivity and specificity for a UNGAL cut-off concentration of $90.5 \mathrm{ng} / \mathrm{mL}$ (Fig. 1A). However, the AuROC value for UNGAL was higher than that for PNGAL in patients with CKD; there was moderate sensitivity and good specificity for a PNGAL cut-off concentration of $504.4 \mathrm{ng} / \mathrm{mL}$ and high sensitivity and $100 \%$ specificity for a UNGAL cut-off concentra-

Table 3. Multivariate logistic regression analyses of risk factors for AKI prediction in AKI high-risk patients $(\mathrm{N}=151)$

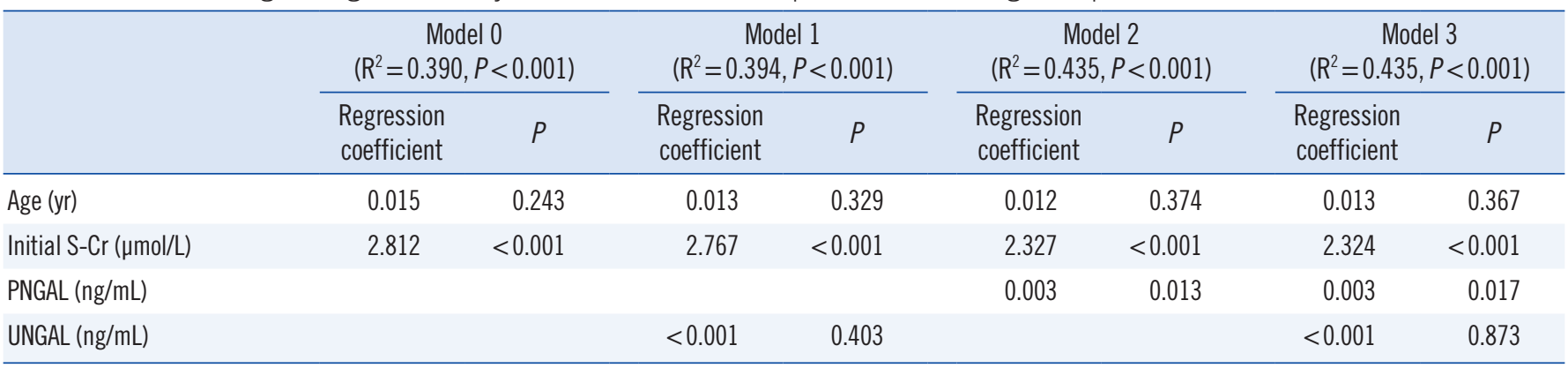

Multivariate logistic regression analysis included variables with univariate $P<0.05$ (age, kidney biomarkers).

Abbreviations: AKI, acute kidney injury; PNGAL, plasma neutrophil gelatinase-associated lipocalin; S-Cr, serum creatinine; UNGAL, urine neutrophil gelatinase-associated lipocalin. 

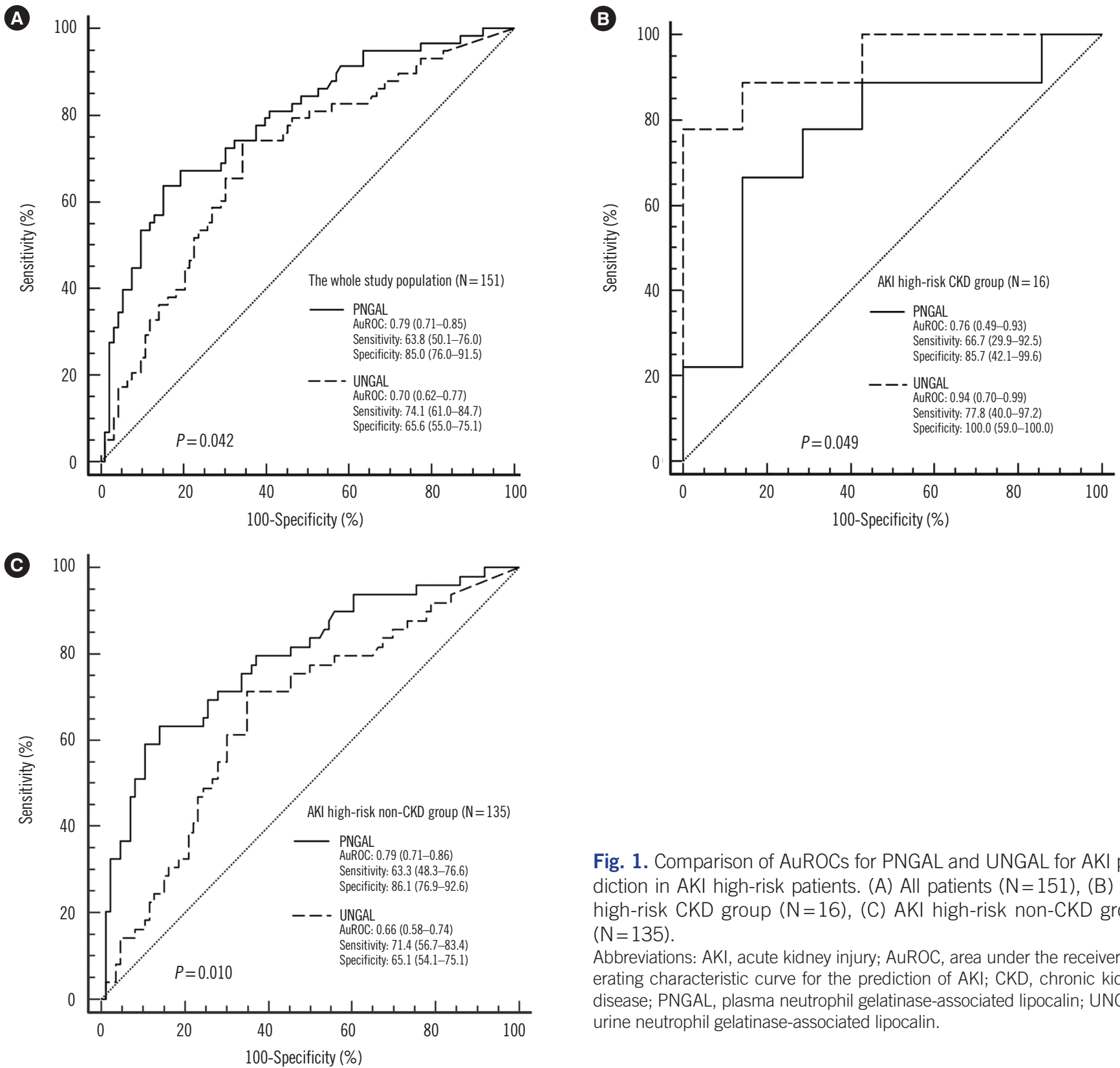

Fig. 1. Comparison of AuROCs for PNGAL and UNGAL for AKI prediction in AKI high-risk patients. (A) All patients ( $N=151$ ), (B) AKI high-risk CKD group $(\mathrm{N}=16)$, (C) AKI high-risk non-CKD group $(\mathrm{N}=135)$.

Abbreviations: AKI, acute kidney injury; AuROC, area under the receiver operating characteristic curve for the prediction of $A K I$; CKD, chronic kidney disease; PNGAL, plasma neutrophil gelatinase-associated lipocalin; UNGAL, urine neutrophil gelatinase-associated lipocalin.

Table 4. Comparison of PNGAL and UNGAL concentrations according to clinical outcomes

\begin{tabular}{|c|c|c|c|c|c|c|c|c|c|}
\hline & \multicolumn{3}{|c|}{ In-hospital mortality } & \multicolumn{3}{|c|}{ ICU admission } & \multicolumn{3}{|c|}{ RRT requirement } \\
\hline & $-^{\dagger}(N=137)$ & $+^{\dagger}(\mathrm{N}=14)$ & $P^{*}$ & $--^{\dagger}(N=110)$ & $+^{\dagger}(\mathrm{N}=41)$ & $P^{*}$ & $-^{\dagger}(\mathrm{N}=147)$ & $+^{\dagger}(\mathrm{N}=4)$ & $P^{*}$ \\
\hline PNGAL (ng/mL) & $\begin{array}{c}206.3 \\
(133.9-351.5)\end{array}$ & $\begin{array}{c}436.6 \\
(152.5-724.7)\end{array}$ & 0.023 & $\begin{array}{c}184.9 \\
(128.3-306.1)\end{array}$ & $\begin{array}{c}387.4 \\
(170.2-513.5)\end{array}$ & $<0.001$ & $\begin{array}{c}211.7 \\
(134.7-360.4)\end{array}$ & $\begin{array}{c}801.5 \\
(543.3-1,071.4)\end{array}$ & 0.003 \\
\hline UNGAL (ng/mL) & $\begin{array}{c}87.0 \\
(26.1-376.6)\end{array}$ & $\begin{array}{c}340.5 \\
(82.6-779.8)\end{array}$ & 0.064 & $\begin{array}{c}76.4 \\
(21.8-315.8)\end{array}$ & $\begin{array}{c}153.3 \\
(51.2-771.9)\end{array}$ & 0.020 & $\begin{array}{c}87.0 \\
(27.7-382.7)\end{array}$ & $\begin{array}{c}2,008.7 \\
(1,004.3-3,327.3)\end{array}$ & 0.003 \\
\hline
\end{tabular}

Data are expressed as median (interquartile range).

${ }^{*} P$ was determined by comparison between patients with and without AKI events using the Mann-Whitney $\mathrm{U}$ test; ${ }^{\dagger}$ - indicates absence of a patient outcome, and + indicates presence of a patient outcome.

Abbreviations: ICU, intensive care unit; PNGAL, plasma neutrophil gelatinase-associated lipocalin; RRT, renal replacement therapy; UNGAL, urine neutrophil gelatinase-associated lipocalin. 
tion of $477.4 \mathrm{ng} / \mathrm{mL}$ (Fig. 1B). The AuROC value in patients without CKD was higher for PNGAL than for UNGAL; there was moderate sensitivity and high specificity for a PNGAL cut-off concentration of $271.0 \mathrm{ng} / \mathrm{mL}$ and moderate sensitivity and specificity for a UNGAL cut-off concentration of $90.5 \mathrm{ng} / \mathrm{mL}$ (Fig. 1C).

The AUROC value of PNGAL was significantly higher than that of UNGAL ( $P=0.042$; Fig. $1 \mathrm{~A})$ in all patients, whereas in the CKD group ( $\mathrm{N}=16)$, AuROC value of UNGAL was significantly higher than that of PNGAL ( $P=0.049$; Fig. 1B). In the AKI highrisk non-CKD group ( $N=135)$, PNGAL showed a significantly higher AuROC value than UNGAL ( $P=0.010$; Fig. 1C).

\section{Comparison of PNGAL and UNGAL concentrations according to clinical outcomes}

PNGAL and UNGAL concentrations differed significantly according to clinical outcomes, such as in-hospital mortality, ICU admission, and RRT requirement (Table 4). Specifically, PNGAL concentrations were higher in patients who died, were admitted to the ICU, and received RRT, and UNGAL concentrations were higher in patients who were admitted to the ICU and/or received RRT.

\section{DISCUSSION}

The main purpose of this study was to evaluate the presence of AKI at admission and to determine the predictive power for AKI development based on S-Cr, PNGAL, and UNGAL concentration measurements in suspected patients. In almost all patients with $\mathrm{AKI}$, except for six (52/58), the condition developed on admission. Soto, et al. [23] reported that PNGAL is an accurate biomarker for the AKI prediction in patients admitted to the emergency department and proposed a three-grade classification of AKI risk based on PNGAL concentration.

The incidence of AKI in this study (58/151, 38.4\%) was higher than that previously reported [19, 20]. Tecson, et al. [19] reported an AKI incidence of $13.5 \%$ in ICU patients, and Egal, et al. [20] reported an AKI incidence of $34.7 \%$ in oliguric critically ill patients. Differences in AKI incidence across study populations could be caused by differences in the constitutions of the patients (AKI risk factors, such as susceptibilities and/or exposures) and/or the clinical judgments of experienced clinicians regarding AKI evaluation. PNGAL, UNGAL, and initial S-Cr concentrations, and initial eGFR all differed significantly between the AKI and non-AKI groups; however, the difference of PNGAL concentration was no longer significant in patients with CKD. This result confirms a strong association of PNGAL and UNGAL concentrations with AKI.

PNGAL and initial S-Cr concentrations emerged as independent predictors of AKI development in AKI high-risk patients based on multivariate regression analysis. However, UNGAL concentration did not show a significant regression coefficient. These findings indicate that AKI was largely determined by the S-Cr concentration, which was already high in the AKI patients due to the characteristics of this population, such as admission to the emergency department and presentation with symptoms that categorized them as AKI high-risk patients. However, the R2 value increased in the model combining PNGAL and UNGAL concentrations with initial S-Cr concentration, reflecting the additive value of NGAL and S-Cr for the AKI prediction.

PNGAL showed similar AuROCs in the AKI high-risk CKD group and AKI high-risk non-CKD group. UNGAL showed a higher AuROC in the AKI high-risk CKD group. In CKD patients, the elimination of NGAL by the kidney is severely deteriorated, while basal PNGAL concentrations tend to be maintained at high concentrations. This leads to a difficulty in discriminating AKI in CKD patients based on PNGAL concentration. Corbacioglu, et al. [24] reported that PNGAL concentrations were higher in AKI patients than in CKD patients. However, in clinical practice, the use of PNGAL concentrations to distinguish between AKI and CKD is limited [24]. Smith, et al. [25] reported that using the UNGALto-urine creatinine ratio in addition to conventional- cardiovascular and kidney risk factors may improve the prediction of disease progression in elderly Caucasian pre-dialysis CKD patients with low-grade proteinuria. Therefore, PNGAL concentration evaluation may be useful as a first step in the AKI prediction in AKI high-risk patients without CKD, whereas UNGAL concentration evaluation may be useful as a first step in the AKI prediction in AKI high-risk patients with CKD. This study provides several cut-off values for PNGAL and UNGAL for these patients and subgroups. The median UNGAL concentration in the AKI highrisk CKD group was significantly higher in AKI patients than in the non-AKI ones. Although the median PNGAL concentration was higher in the AKI group than in the non-AKI group, the difference was not statistically significant.

PNGAL and UNGAL concentrations could also reflect patient outcomes in AKI high-risk patients. Several studies identified both PNGAL and UNGAL concentrations as predictors of mortality or diagnostic markers for AKI in critically ill patients or patients who underwent cardiac surgery with cardiopulmonary bypass [26-29]. However, these studies excluded CKD patients at the patient selection step or concluded that the discriminative performance of both PNGAL and UNGAL concentrations was 
reduced by preexisting CKD. By contrast, we included CKD patients as a subgroup in this study and identified that UNGAL concentration evaluation may be useful as a first step for AKI prediction in AKI high-risk patients with CKD.

The present study has several limitations. The definition for AKI was extended because S-Cr records in the seven days before UNGAL measurement were not available for most patients. In addition, urine output criteria were excluded in the determination of AKI. The median initial S-Cr concentration in the AKI group was high, at $117.6 \mu \mathrm{mol} / \mathrm{L}$, because kidney injury had already occurred in most patients who visited the emergency department. In addition, 16 out of the 151 patients had CKD. Therefore, further studies should be performed with a larger number or various populations of patients. Finally, we used eGFR values for the determination of AKI and CKD instead of measured GFR values. GFR measurement remains a significant confirmatory test for reduced eGFR [30]. However, all GFR measurement methods are imprecise and could be biased against true GFR [30]. Standardization and calibration among methods, with the development of more accurate eGFR equations may provide helpful data for both clinical work and research [30].

In conclusion, PNGAL may serve as a useful biomarker for AKI prediction in AKI high-risk patients. However, in AKI highrisk patients with CKD, UNGAL showed better AKI prediction power than PNGAL. This approach could be beneficial for the timely and optimized management of AKI in AKI high-risk patients with or without CKD. Our findings may guide in selecting the most appropriate specimens for NGAL assays based on the presence of CKD in AKI high-risk patients. The current trend in NGAL studies is focused on evaluating the potential role of NGAL in specific study populations, such as patients with decompensated cirrhosis, diabetic kidney disease, heart failure with or without left ventricular assisted device implantation; infants with congenital urinary tract obstruction; and patients with abdominal aortic aneurysm [31-35]. Further studies are needed to investigate the role of NGAL as a biomarker of AKI in specific patient groups.

\section{AUTHOR CONTRIBUTIONS}

AY designed the study, analyzed the data, and wrote the draft; $\mathrm{CHL}$ and YMY conceived the study and finalized the draft; HK, HWM, and $\mathrm{MH}$ reviewed the draft and commented on it. All authors have accepted responsibility for the entire content of this manuscript and approved submission.

\section{CONFLICTS OF INTEREST}

The authors report no conflicts of interest. The authors alone are responsible for the content and writing of the paper.

\section{RESEARCH FUNDING}

This study was supported by the grant and product from Bioporto Diagnostics A/S.

\section{ORCID}

Ahram Yi

Chang-Hoon Lee

https://orcid.org/0000-0002-3107-936X

Yeo-Min Yun

https://orcid.org/0000-0002-5402-2681

Hanah Kim

https://orcid.org/0000-0002-5485-8331

https://orcid.org/0000-0002-3266-638X

Hee-Won Moon

Mina Hur

https://orcid.org/0000-0001-9509-6073

https://orcid.org/0000-0002-4429-9978

\section{REFERENCES}

1. KDIGO AKI guideline work group. KDIGO clinical practice guideline for acute kidney injury. Summary of recommendation statements. Kidney Int Suppl 2012;2:8-12.

2. Thomas ME, Blaine C, Dawnay A, Devonald MA, Ftouh S, Laing C, et al. The definition of acute kidney injury and its use in practice. Kidney Int 2015;87:62-73.

3. Schetz M, Gunst J, Van den Berghe G. The impact of using estimated GFR versus creatinine clearance on the evaluation of recovery from acute kidney injury in the ICU. Intensive Care Med 2014;40:1709-17.

4. Doi K, Yuen PS, Eisner C, Hu X, Leelahavanichkul A, Schnermann J, et al. Reduced production of creatinine limits its use as marker of kidney injury in sepsis. J Am Soc Nephrol 2009;20:1217-21.

5. Lopes JA, Jorge S, Resina C, Santos C, Pereira A, Neves J, et al. Acute kidney injury in patients with sepsis: a contemporary analysis. Int J Infect Dis 2009;13:176-81.

6. Khawaja S, Jafri L, Siddiqui I, Hashmi M, Ghani F. The utility of neutrophil gelatinase-associated lipocalin (NGAL) as a marker of acute kidney injury (AKI) in critically ill patients. Biomark Res 2019;7:4.

7. Kashani K, Macedo E, Burdmann EA, Hooi LS, Khullar D, Bagga A, et al. Acute kidney injury risk assessment: differences and similarities between resource-limited and resource-rich countries. Kidney Int Rep 2017;2:519-29.

8. Mishra J, Mori K, Ma Q, Kelly C, Barasch J, Devarajan P. Neutrophil gelatinase-associated lipocalin: a novel early urinary biomarker for cisplatin nephrotoxicity. Am J Nephrol 2004;24:307-15.

9. Mori K and Nakao K. Neutrophil gelatinase-associated lipocalin as the real-time indicator of active kidney damage. Kidney Int 2007;71:96770.

10. Hirsch R, Dent C, Pfriem H, Allen J, Beekman RH 3rd, Ma Q, et al. NGAL is an early predictive biomarker of contrast-induced nephropathy in children. Pediatr Nephrol 2007;22:2089-95.

11. Albert C, Haase M, Albert A, Kropf S, Bellomo R, Westphal S, et al. Urinary biomarkers may complement the Cleveland score for prediction of 
adverse kidney events after cardiac surgery: A pilot study. Ann Lab Med 2020;40:131-41.

12. Cho SY and Hur M. Hepcidin and neutrophil gelatinase-associated lipocalin as a biomarker for acute kidney injury linked iron metabolism. Ann Lab Med 2020;40:97-8.

13. Cavalier E, Bekaert AC, Carlisi A, Legrand D, Krzesinski JM, Delanaye P. Neutrophil gelatinase-associated lipocalin (NGAL) determined in urine with the Abbott Architect or in plasma with the Biosite Triage? The laboratory's point of view. Clin Chem Lab Med 2011;49:339-41.

14. Suzuki M, Wiers KM, Klein-Gitelman MS, Haines KA, Olson J, Onel KB, et al. Neutrophil gelatinase-associated lipocalin as a biomarker of disease activity in pediatric lupus nephritis. Pediatr Nephrol. 2008;23:40312.

15. Kim SY, Jeong TD, Lee W, Chun S, Sung S, Kim SB et al. Plasma neutrophil gelatinase-associated lipocalin as a marker of tubular damage in diabetic nephropathy. Ann Lab Med 2018;38:524-9.

16. Filiopoulos V, Biblaki D, Lazarou D, Chrisis D, Fatourou M, Lafoyianni S, et al. Plasma neutrophil gelatinase-associated lipocalin (NGAL) as an early predictive marker of contrast-induced nephropathy in hospitalized patients undergoing computed tomography. Clin Kidney J 2013;6:57883.

17. Tuladhar SM, Püntmann VO, Soni M, Punjabi PP, Bogle RG. Rapid detection of acute kidney injury by plasma and urinary neutrophil gelatinase-associated lipocalin after cardiopulmonary bypass. J Cardiovasc Pharmacol 2009;53:261-6.

18. Zampini G, De Berardinis B, Di Somma S. Urinary versus plasma NGAL. In: Hur M and Di Somma S, eds. Neutrophil gelatinase-associated lipocalin: from laboratory to clinical utility. First edition. New York: Nova Biomedical, 2014:49-53.

19. Tecson KM, Erhardtsen E, Eriksen PM, Gaber AO, Germain M, Golestaneh L, et al. Optimal cut points of plasma and urine neutrophil gelatinase-associated lipocalin for the prediction of acute kidney injury among critically ill adults: retrospective determination and clinical validation of a prospective multicentre study. BMJ Open 2017;7:e016028.

20. Egal M, de Geus HR, Groeneveld AB. Neutrophil gelatinase-associated lipocalin as a diagnostic marker for acute kidney injury in oliguric critically ill patients: a post-hoc analysis. Nephron 2016;134:81-8.

21. NGAL Meta-Analysis Investigator Group, et al. Neutrophil gelatinase-associated lipocalin measured on clinical laboratory platforms for the prediction of acute kidney injury and the associated need for dialysis therapy: a systematic review and meta-analysis. Am J Kidney Dis. $2020 \mathrm{Jul}$ 14:S0272-6386(20)30780-0.

22. Kellum JA, Bellomo R, Ronco C. Does this patient have acute kidney injury? An AKI checklist. Intensive Care Med 2016;42:96-9.

23. Soto K, Papoila AL, Coelho S, Bennett M, Ma Q, Rodrigues B, et al. Plasma NGAL for the diagnosis of AKI in patients admitted from the emergency department setting. Clin J Am Soc Nephrol 2013;8:205363.

24. Corbacıoglu SK, Cevik Y, Akinci E, Uzunosmanoglu H, Dagar S, Safak T, et al. Value of plasma neutrophil gelatinase-associated lipocalin (NGAL) in distinguishing between acute kidney injury (AKI) and chronic kidney disease (CKD). Turk J Emerg Med 2017;17:85-8.

25. Smith ER, Lee D, Cai MM, Tomlinson LA, Ford ML, McMahon LP, et al. Urinary neutrophil gelatinase-associated lipocalin may aid prediction of renal decline in patients with non-proteinuric Stages 3 and 4 chronic kidney disease (CKD). Nephrol Dial Transplant 2013;28:1569-79.

26. Aydoğdu M, Gürsel G, Sancak B, Yeni S, Sarı G, Taşyürek S, et al. The use of plasma and urine neutrophil gelatinase associated lipocalin (NGAL) and cystatin C in early diagnosis of septic acute kidney injury in critically ill patients. Dis Markers 2013;34:237-46.

27. Matsa R, Ashley E, Sharma V, Walden AP, Keating L. Plasma and urine neutrophil gelatinase-associated lipocalin in the diagnosis of new onset acute kidney injury in critically ill patients. Crit Care 2014;18:R137.

28. Schley G, Köberle C, Manuilova E, Rutz S, Forster C, Weyand M, et al. Comparison of plasma and urine biomarker performance in acute kidney injury. PLoS One 2015;10:e0145042.

29. Mahmoodpoor A, Hamishehkar H, Fattahi V, Sanaie S, Arora P, Nader ND. Urinary versus plasma neutrophil gelatinase-associated lipocalin (NGAL) as a predictor of mortality for acute kidney injury in intensive care unit patients. J Clin Anesth 2018;44:12-7.

30. Levey AS and Inker LA. GFR as the "Gold Standard": estimated, measured, and true. Am J Kidney Dis 2016;67:9-12.

31. Huelin P, Solà E, Elia C, Solé C, Risso A, Moreira R, et al. Neutrophil gelatinase-associated lipocalin for assessment of acute kidney injury in cirrhosis: a prospective study. Hepatology 2019;70:319-33.

32. Siddiqui K, AI-Malki B, George TP, Nawaz SS, Rubeaan KA. Urinary Nacetyl-beta-d-glucosaminidase (NAG) with neutrophil gelatinase-associated lipocalin (NGAL) improves the diagnostic value for proximal tubule damage in diabetic kidney disease. 3 Biotech 2019;9:66.

33. Grosman-Rimon L, Hui SG, Freedman D, Elbaz-Greener G, Cherney D, Rao V. Biomarkers of inflammation, fibrosis, and acute kidney injury in patients with heart failure with and without left ventricular assist device implantation. Cardiorenal Med 2019;9:108-16.

34. Kostic D, Dos Santos Beozzo GPN, do Couto SB, Kato AHT, Lima L, Palmeira $\mathrm{P}$, et al. First-year profile of biomarkers for early detection of renal injury in infants with congenital urinary tract obstruction. Pediatr Nephrol 2019;34:1117-28.

35. Groeneveld ME, Struik JA, Musters RJP, Tangelder GJ, Koolwijk P, Niessen HW, et al. The potential role of neutrophil gelatinase-associated lipocalin in the development of abdominal aortic aneurysms. Ann Vasc Surg 2019;57:210-9. 